\title{
The application of probability neural network in remote sensing image processing based on $k$-means
}

\author{
Min $\mathrm{Hu}$ and Feng-Jun Li* \\ School of Mathematics and Statistics, Ningxia University, Yinchuan, China \\ E-mail:fjli@nxu.edu.cn \\ *Corresponding author
}

\begin{abstract}
In this paper, we design an approach which is a combination of $\mathrm{k}$-means clustering and probability neural network method to classify the remote sensing image. The proposed method allows the implementation of Kaufman approach to get clustering centers, which are used as initial centers in $\mathrm{k}$-means algorithm. Then the image is divided into $\mathrm{k}$ number of clusters by using the k-means algorithm. Finally, the pixels are divided into k classes according to probability neural network. The results indicate that the classified image is consistent with the original image and all kinds of characteristics are relatively well preserved.
\end{abstract}

Keywords: Image Classification; Kaufman Approach; K-Means Clustering; Probability Neural Network.

\section{Introduction}

Remote sensing image classification is an important part, as well as one of the broadest areas of remote sensing digital image processing. The process can be described as extracting the image feature information and classifying based on the acquired characteristics. Its purpose is to divide the each pixel into different categories based on some rules or algorithms according to the spectral brightness, space structure or other features[1]. Image classification generally can be divided into supervised and unsupervised classification according to the degree of human intervention[1-3]. The supervised classification tends to have higher classification accuracy but the influence of human factors is stronger. The unsupervised classification has large blindness and the calculation speed is too slow to achieve the ideal classification result.

The paper is organized as follows. First, a preliminary classification is conducted by k-means. Afterwards, trains the PNNs according to the training samples and classifies all pixel points by using trained neural network. Finally, a new remote sensing image is resynthesized by using classified pixel points. 


\section{The Probabilistic Neural Networks Model}

The Probabilistic Neural Network (PNN) was first proposed by D. F. Specht in the 1990[1,2]. The PNN, based on RBF neural network, fuses density estimation and Bayes decision rule, and has more significant advantages especially in terms of pattern classification. According to Bayes decision rule: Suppose that there are $c$ classes classification problems $w_{1}, w_{2}, \cdots, w_{c}$, for a given input vector $x=\left[x_{1}, x_{2}, \cdots, x_{n}\right]$, the basic principle of the classification is as follows:

$$
\text { if } p\left(w_{i} \mid x\right)>p\left(w_{j} \mid x\right), \forall j \neq i \text { then } x \in w_{i}, \quad i=1,2, \cdots, c
$$

where $p\left(w_{i} \mid x\right)=p\left(w_{i}\right) p\left(x \mid w_{i}\right) / p(x)$. The probability density function $p\left(w_{i} \mid x\right)$ is unknown, and the Parzen estimation by Gaussian kernel is as follows:

$$
p\left(x \mid w_{i}\right)=\frac{1}{N_{i}} \sum_{k=1}^{N_{i}} \frac{1}{(2 \pi)^{\frac{l}{2}} \sigma^{l}} \exp \left(-\frac{\left\|x-x_{i k}\right\|^{2}}{2 \sigma^{2}}\right)
$$

where the k-th training sample ${ }^{x_{i k}}$ belongs to ${ }^{w_{i}}, l$ is the dimension, $\sigma$ is smoothing parameter, and $N_{i}$ is the total number of training samples in ${ }^{w_{i}}$.

Removing the common elements, the discriminant can be simplified to:

$$
g_{i}(x)=\frac{p\left(w_{i}\right)}{N_{i}} \sum_{k=1}^{N_{i}} \exp \left(-\frac{\left\|x-x_{i k}\right\|^{2}}{2 \sigma^{2}}\right)
$$

And then all samples are normalized:

$$
\sum_{i=1}^{l} x_{i}^{2}=1, \square x-x_{i k} \square^{2}=\square x \square^{2}+\square x_{i k} \square^{2}-2 x^{\prime} x_{i k}=2-2 x^{\prime} x_{i k}
$$

Thus,

$$
\text { if } g_{i}(x)>g_{j}(x) \quad \forall j \neq i \text {, then } x \in w_{i}
$$

The network structure of PNNs mainly consists of three layers: an input layer, a pattern layer(hidden layer) and a competitive output layer. The structure of PNNs is shown in Fig.1. PNN is commonly used as supervised classifier in various applications. Foody[3]proved that PNN was able to accurately map land cover for an agricultural site better than other networks like Multilayer Perceptron (MLP) and RBFNN. 


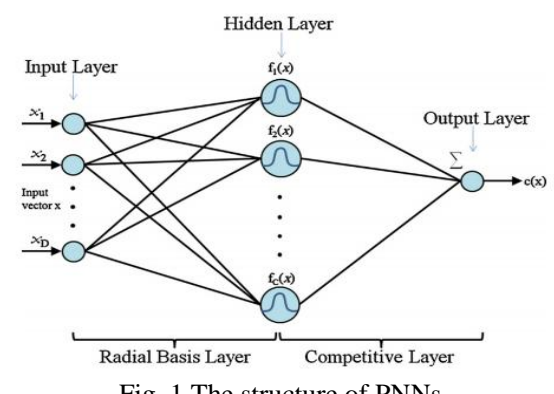

Fig. 1 The structure of PNNs

\section{K-Means Clustering Algorithm}

Let us consider an image with resolution of $x \times y$ and the image has to be clustered into $\mathrm{k}$ clusters. Let $p(x, y)$ be an input pixel to be clustered and $c_{k}$ are the cluster centers. The algorithm for k-means[3,4]clustering is as below:

Initialize number of cluster $\mathrm{k}$ and centers.

For each pixel, the Euclidean distance $d=\square p(x, y)-c_{k} \square$ is calculated between the center and each pixel of an image. Assign all the pixels to the nearest center based on distance $d$.

After all pixels have been assigned, recalculate new position of the center using the relation given below:

$$
c_{k}=\frac{1}{k} \sum_{y \in c_{k}} \sum_{x \in c_{k}} p(x, y) .
$$

Repeat the process until it satisfies the tolerance or error value. Reshape the cluster pixels into image.

The k-means is easy to realize but there are also some limitations. The ultimate clustering result depends on initial center. There are many initialization methods[3,4], as Forgy's approach, Macqueen's approach, Kaufman approach and so on. Kaufman method is the most stable method and obtains the best classification result compared with other initialization methods. Here Kaufman approach is selected to find the optimal data point to define a cluster center.

\section{Simulation Experiment and Results}

In the paper, the proposed algorithm is divided into three parts. First the cluster center is generated by Kaufman approach. This center is used as initial center in $\mathrm{k}$-means algorithm. The image is divided into $\mathrm{k}$ number of clusters by $\mathrm{k}$-means algorithm. Finally, the pixels are divided into $\mathrm{k}$ classes according to PNNs. 
Step 1: Load the image to be reduced aptly and display the reduced image, as shown in Fig. 2.

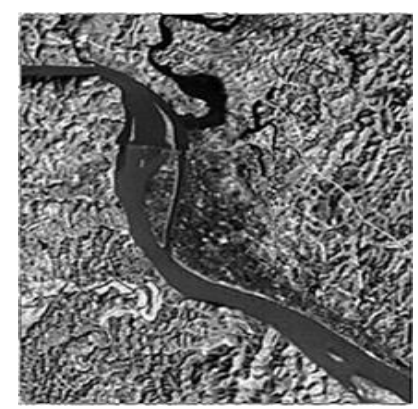

Fig. 2 The reduced image

Step 2: Convert image data to the training data. There are two aspects need to be noticed. One is that convert unit8 structure into double type. The other one is each row in the data matrix is a sample and each column as a property.

Step 3: The pixels are classified by utilizing the k-means cluster analysis. Here we have taken the number of clusters, $k=3$ are used as initial center in the k-means clustering algorithm. And the clustering result is shown in Fig. 3.
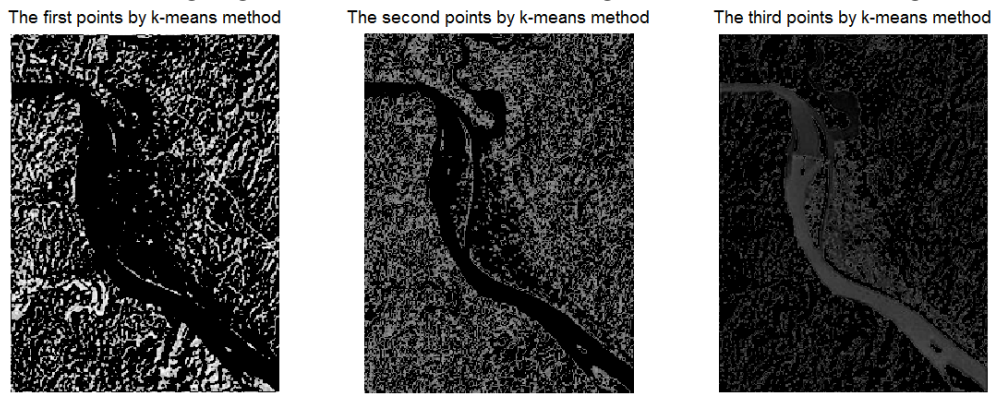

Fig. 3 The clustering results by k-means method

Step 4: Select part of the samples as the training sample of PNN based on k-means clustering result. Note here that the median value belongs to the same kind of color as representative value of this kind of color.

Step 5: PNN model is constructed based on sample data. Here newpnn function in Matlab toolbox can be used to construct a PNN, ind2vec function can convert the desired category into vector, and vec2ind function convert the output vector into pointer.

Step 6: Check the network classification results through back substitution 
sample data, the result is shown in Fig.4. As can be seen from Fig. 4, the sample data are well approximated by neural network.

Step 7: Classify the remote sensing image according to the PNN, and the classification result is shown in Fig. 5.
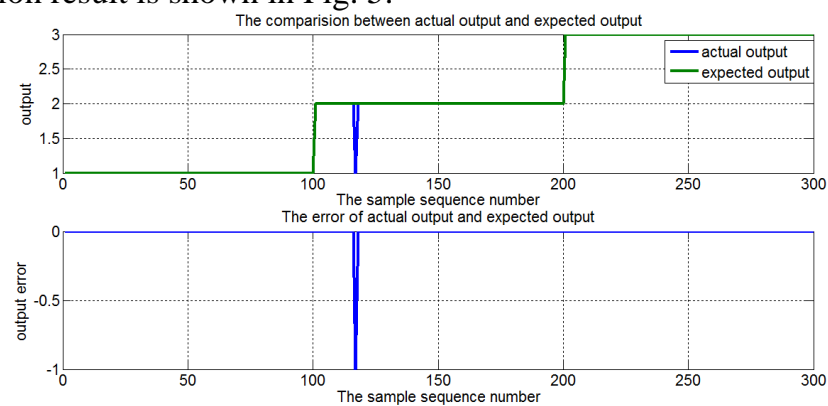

Fig. 4 The network classification results and error curve

More precisely, the results of classification accuracy and Kappa coefficient are specifically revealed in Table 1 . The overall accuracy obtained in the proposed method is $89.94 \%$ with a Kappa coefficient of 0.8513 . The target classification has been already obtained effectively. Combining the two methods result in higher classification accuracy when compared with using k-means method and PNN alone. It shows the effectiveness of the proposed method.

Tab. 1 The caparison of classification precision

\begin{tabular}{ccc}
\hline Classification method & Classification accuracy & Kappa coefficient \\
\hline The k-means method & $81.12 \%$ & 0.7001 \\
The PNN method & $70.05 \%$ & 0.6112 \\
The proposed method & $89.94 \%$ & 0.8513 \\
\hline & &
\end{tabular}

Fig. 5 The classification result by using PNN 


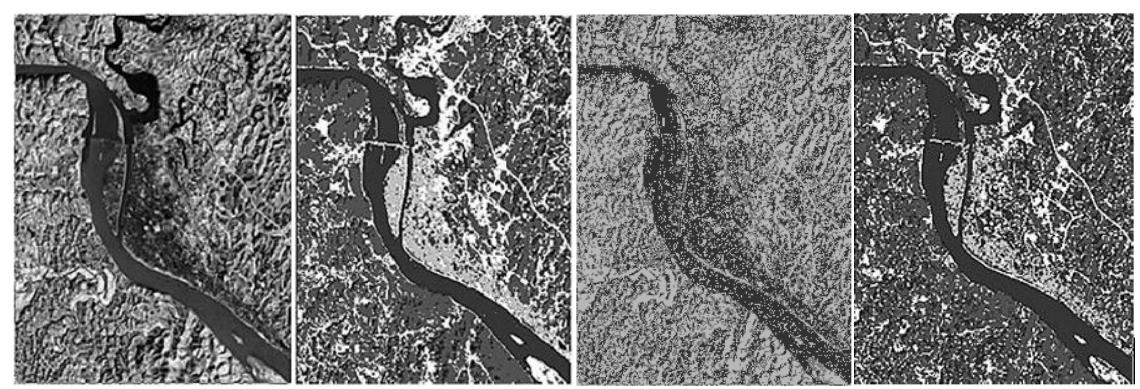

(a) The Original image (b) The Proposed method (c) The PNN method (d) The k-means method Fig. 6 The classification results by different methods

\section{Conclusion}

This paper proposes a method combined k-means with PNN method. The method not only exerts the advantages of k-means but also maintains the self-organization characteristic of PNN algorithm. What's more, it is further proved that the classification precision of the proposed method is better than the traditional k-means and PNN methods and it is to be more effective in the classification of remote sensing data.

\section{Acknowledgments}

This work was supported by the National Natural Science Foundation of China (Grant Nos. 11261042 and 61662060).

\section{References}

1. Y. T. Qian, M. C. Ye, and J. Zhou, Hyper-spectral image classification based on structured sparse logistic regression and three dimensional wavelet texture features, IEEE Transaction on Geoscience and Remote Sensing, vol. 51, no. 4, pp. 2276-2291, 2013.

2. J. Sun, J. Yang, and J. Qu, Automatic remotely sensed image classification in a grid environment based on the maximum likelihood method, Mathematic Computing Model, vol. 58, no. 3-4, pp. 573-581, 2013.

3. M. Han and W. Yao, Remote sensing image classification based on neural network algorithm, Neurocomputing, vol. 21, no. 3, pp. 377-386, 2012.

4. Y. Shao and R. S. Lunetta, Comparison of support vector machine, neural network, and CART algorithms for the land-cover classification using 
limited training data points, ISPRS Journal of Photogrammetry and Remote Sensing, vol. 70, pp. 78-87, 2012.

5. D.F.Specht, Probabilistic neural networks, Neural Networks, vol. 3, no. 1, pp. 109-118, 1990.

6. D.F. Specht, Probabilistic neural networks for classification, mapping, or associative memory, Neural Networks, vol. 1, pp. 525-532, 1988.

7. J. Lounousse and A. E. Motassadeq, Using an unsupervised approach of probabilistic neural network for land use classification from multitemporal satellite images, Applied Soft Computing, vol. 30, pp. 1-13, 2015.

8. H. Yao, Q. L. Duan, and D. L. Li, An improved k-means clustering algorithm for fish image segmentation, Mathematical and Computer Modelling, vol. 58, no. 3-4, pp.790-798, 2013.

9. Y. M. Cheung, K-means: A new generalized k-means clustering algorithm, Pattern Recognition Letters, vol. 24, no. 15, pp. 2883-2893, 2003.

10. M. E. Celebi, H. A. Kingravi, and P. A. Vela, A comparative study of efficient initialization methods for the k-means clustering algorithm, Expert Systems with Applications, vol. 40, no. 1, pp. 200-210, 2013.

11. R. Duwairi and M. A. Rahmeh, A novel approach for initializing the spherical k-means clustering algorithm, Simulation Modelling Practice and Theory, vol. 54, pp. 49-63, 2015. 